\title{
Diseño de aeronave de combate no tripulada de quinta generación para el apoyo aéreo cercano
}

| Fecha de recibido: 2 de marzo del 2021 | Fecha de aprobación: 8 de abril del 2021 |

\section{Esteban \\ Morales Aguirre}

Estudiante de Ingeniería Aeronáutica

Universidad Pontificia Bolivariana Colombia

Semillero de Investigación en Ingeniería Aeroespacial Rol de investigador: teórico, experimental y escritura http://orcid.org/0000-0001-7054-7376

凶estanimation1@hotmail.com

\section{Daniel Enrique Aldana Lopera}

Estudiante de Ingeniería Aeronáutica

Universidad Pontificia Bolivariana Colombia

Semillero de Investigación en Ingeniería Aeroespacial Rol de investigador: teórico, experimental y escritura https://orcid.org/0000-0003-1351-6025 $\triangle$ danielenrique.aldana@mail.polimi.it

\section{Mateo \\ Duarte García}

Estudiante de maestría en Energía

Universidad Pontificia Bolivariana

Colombia

Semillero de Investigación en Ingeniería Aeroespacial Rol de investigador: teórico, experimental y escritura https://orcid.org/0000-0002-9926-3104

$\square$ mateoduartegarcia@yahoo.es

\section{Javier Mauricio Sabogal Jaramillo}

Estudiante de Ingeniería Aeronáutica

Universidad Pontificia Bolivariana

Colombia

Semillero de Investigación en Ingeniería Aeroespacial Rol de investigador: teórico, experimental y escritura https://orcid.org/0000-0003-3625-0709

$\triangle$ javier.sabogal@upb.edu.co

\section{Paniagua García \\ Esteban}

Estudiante de Ingeniería Aeronáutica

Universidad Pontificia Bolivariana

Colombia

Semillero de Investigación en Ingeniería Aeroespacial Rol de investigador: teórico, experimental y escritura https://orcid.org/0000-0003-1734-1736

$\bowtie$ esteban.paniaguag@upb.edu.co

\section{Jorge Elias Montoya Vélez \\ Docente de cátedra}

Universidad Pontificia Bolivariana Colombia

Semillero de Investigación en Ingeniería Aeroespacial

Rol de investigador: escritura https://orcid.org/0000-0002-0006-7841

$\bowtie$ jorgeelias.montoya@upb.edu.co

\section{Juan Pablo \\ Alvarado Perilla \\ Docente de tiempo completo \\ Universidad Pontificia Bolivariana}

Colombia

Semillero de Investigación en Ingeniería Aeroespacial

Rol de investigador: teórico y escritura https://orcid.org/0000-0001-5257-6093

$\triangle$ juan.alvarado@upb.edu.co

Cómo citar este artículo: Morales Aguirre, E., Duarte García, M., Paniagua García, E., Aldana Lopera, D. E., Sabogal Jaramillo,

J. M., Montoya Vélez, J. E., \& Alvarado Perilla, J. P. (2021). Diseño de aeronave de combate no tripulada de quinta generación para el apoyo aéreo cercano. Ciencia y Poder Aéreo, 16(1), 55-74, https://doi.org/10.18667/cienciaypoderaereo.693 


\section{Diseño de aeronave de \\ combate no tripulada
de quinta generación para
el apoyo aéreo cercano \\ combate no tripulada
de quinta generación para
el apoyo aéreo cercano \\ combate no tripulada
de quinta generación para
el apoyo aéreo cercano}

\section{Design of a Fifth Generation Unmanned Fighter Aircraft for Close Air Support}

Resumen: La evolución en el diseño de aeronaves de combate se ha visto modificada por la inclusión de nuevos parámetros de alta exigencia, dentro de los cuales se incluyen las áreas de investigación relacionadas con la aerodinámica, la furtividad y la optimización en los procesos de operación. El presente artículo contempla el diseño de un Vehículo de Combate Aéreo no Tripulado (UCAV) para la ejecución de misiones de Apoyo Aéreo Cercano (CAS por sus siglas en inglés) en las próximas décadas. Los resultados obtenidos demuestran las habilidades de los UCAV como aeronaves de quinta generación para el reemplazo de flotas reconocidas mundialmente (A-10 Thunderbolt II y Sukhoi Su-25) y, además, garantiza su utilidad y viabilidad en los futuros entornos de combate. Así mismo, la investigación se enfoca en una de las variables de mayor discusión respecto a la supervivencia en el combate aéreo, se trata de la furtividad por fenómenos electromagnéticos, con la cual se obtuvieron valores de Sección Equivalente de Radar (RCS) iguales a -24,18 dBsm o representables en un área de detectabilidad de 0,0038 $\mathrm{m}^{2}$ en configuración limpia, de modo que este valor es inferior al de aeronaves furtivas como lo es el Northrop Grumman B-2 Spirit. Finalmente, el diseño permite la operación con un máximo peso de despegue de $61,900 \mathrm{lb}$ y una carga paga de $11,240 \mathrm{lb}$ que se acondicionan a una configuración alar y de estabilizadores para rangos transónicos.

Palabras clave: aeronave no tripulada; apoyo aéreo cercano; furtividad; militar.

Abstract: The evolution of combat aircraft design has been modified by the inclusion of new highly demanding parameters within the research areas of aerodynamics, stealth, and operating processes optimization. Hence, this article presents the design of an unmanned combat aerial vehicle (UCAV) for the execution of close air support (CAS) missions in the forthcoming decades. The results achieved prove the abilities of UCAVs as fifth-generation aircrafts for the replacement of internationally recognized air fleets (A-10 Thunderbolt II and Sukhoi Su-25) and, likewise, ensure their usefulness and feasibility in future combat environments. This research focuses on one of the most widely discussed variables in air combat survival, that is, stealth due to electromagnetic phenomena, for which we obtained a radar cross-section (RCS) value of $-24.18 \mathrm{dBsm}$, represented in a detectability area of $0.0038 \mathrm{~m}^{2}$ in clean configuration, that is lower than the reported by stealth aircrafts such as the Northrop Grumman B-2 Spirit. The design of the proposed aircraft allows operation with a maximum takeoff weight of $61,900 \mathrm{lbs}$. and a maximum payload of 11,240 lbs., retrofitted to a wing and outrigger configuration for transonic ranges.

Keywords: Unmanned aircraft; close air support; stealth; military.

Resumo: A evolução na concepção de aeronaves de combate foi modificada pela inclusão de novos parâmetros de alta exigência, incluindo as áreas de investigação relacionadas com a aerodinâmica, a furtividade e a otimização dos processos operacionais. 0 presente artigo contempla o desenho de um Veículo de Combate Aéreo Não Tripulado (UCAV) para a execução de missões de Apoio Aéreo Próximo (CAS) nas próximas décadas. Os resultados obtidos demonstram as capacidades dos UCAV como aeronaves de quinta geração para a substituição de frotas reconhecidas a nível mundial (A-10 Thunderbolt Il e Sukhoi Su-25) e, além disso, garante sua utilidade e viabilidade nos futuros ambientes de combate. Da mesma forma, a pesquisa se concentra em uma das variáveis de maior discussão a respeito da sobrevivência no combate aéreo, trata-se da furtividade por fenômenos eletromagnéticos, com a qual se obtiveram valores de Seção Equivalente de Radar (RCS) iguais a -24,18 dBsm ou representáveis numa área de detectabilidade de $0,0038 \mathrm{~m}^{2}$ em configuração limpa, de modo que este valor é inferior ao de aeronaves furtivas como é o Northrop Grumman B-2 Spirit. Finalmente, o projeto permite a operação com peso de descolagem máximo de 61,900 lb e uma carga paga de 11,240 lb que sejam condicionados a uma configuração alar e de estabilizadores para escalas transônicas.

Palavras-chave: aeronave não tripulada; apoio aéreo aproximado; furtividade; militar. 
Las múltiples estrategias utilizadas por las fuerzas militares en conflictos armados han sido causantes de la creación de aeronaves con capacidades únicas para el cumplimiento de tareas especializadas y con un alto margen de éxito en su operación. Esto ha permitido la constante evolución del sector aeronáutico-militar, pues se han propuesto diseños enfocados a misiones específicas, por ejemplo, una de las más famosas se denomina Patrulla Aérea de Combate (CAP, por sus siglas en inglés), la cual define una aeronave con capacidad de superioridad aérea, en otras palabras, configuración aire-aire con el propósito de patrullaje y seguridad del espacio aéreo (Dirwan, 2020). Otro ejemplo es la existencia de misiones de ataque donde se da el uso de bombas o misiles aire-tierra para objetivos terrestres, una práctica catalogada como bombardeo de precisión -bombas inteligentes guiadas por láser, programadas por medio de coordenadas- (Andrew et al., 2018).

El análisis del avance tecnológico de los últimos años establece que se debe tener en cuenta la combinación de las misiones, lo que es consecuente con la aparición de las cazas polivalentes o Avión de Combate Polivalente (MRCA por sus siglas en inglés), como lo son el F-16 Fighting Falcon o el F/A-18 Hornet (Biswas, 2019). Sin embargo, una de las misiones más conocidas en la aviación militar mundial es el Apoyo Aéreo Cercano (CAS, por sus siglas en inglés), este efectúa operaciones relacionadas con el ataque y supresión de enemigos con la finalidad de apoyar tropas terrestres en la zona de combate. Estas consideraciones son convenientes para el diseño de aeronaves como el A-10 Thunderbolt II, Sukhoi SU-25, North American Rockwell OV-10 Bronco y el Embraer EMB 314 Super Tucano que se especializan en el uso de armas como misiles aire-tierra, bombas y proyectiles a baja cota, convirtiéndose en una extensión del campo de batalla terrestre, lo que tiene altas implicaciones morales en las unidades de tierra (Correll, 2019).

Actualmente, destaca el uso de aeronaves no tripuladas para misiones de ataque de precisión como el MQ-9 Reaper (Watts, 2013). Esta tecnología está sometida a constantes análisis, pues, aunque los pilotos no se encuentran en peligro real durante la misión, se necesita superar varios desafíos en materia de confiabilidad (Fields, 2012). De acuerdo con lo anterior, se han investigado los métodos más comunes de hackeo de este tipo de aeronaves, para así concretar estrategias que superen los ciberataques. También se demuestra que los ataques pueden generar daños irreversibles en la aeronave y la pérdida total del control (Rani et al., 2016). Asimismo, estos vehículos enfrentan armas de mitigación e interferencia que pretenden derribar drones; dichas armas ya han sido investigadas y se han sugerido pasos a seguir para eliminar las incursiones de aeronaves no tripuladas en espacio aéreo restringido (Sathyamoorthy, 2015).

Sin embargo, las limitaciones y amenazas de los drones se han documentado de forma minuciosa; incluso, se han hecho investigaciones en las que se simulan situaciones de estos ataques hacia drones con el fin de estandarizar prácticas de operación confiables (Yaacoub et al., 2020). Además, gracias a las investigaciones y evoluciones obtenidas en los últimos años, se puede considerar a las aeronaves no tripuladas como el futuro de la aviación; prueba de esto es el desarrollo de nuevos UCAv por parte de entidades entre ellas la OTAN - que apoyan la investigación para el diseño de aeronaves no tripuladas con características furtivas como el Saccon, con objetivos de estudio de estabilidad y control de estas configuraciones (Cummings \& Schütte, 2012). De ese modo, estos diseños son usados después para el desarrollo de un UCAV funcional, lo que muestra una constante evolución de los avances científicos en esta materia (Cummings \& Schütte, 2012).

Por otra parte, el diseño de aeronaves se encuentra acotado bajo una categorización de generaciones que consideran la evolución en el campo de operaciones, por lo tanto, a medida que se fortalecen las formas de mitigar la operatividad de una aeronave, deberá existir un desarrollo que contrarreste, recupere y supere la operatividad anterior. La asociación estadounidense Atlantic Council establece que la quinta generación posee una amplia gama de armas de precisión acompañadas por sistemas de apuntado electroópticos, capacidades de guerra electrónica-interferencia de radares-, capacidades de 
comunicación -enlaces de datos de baja probabilidad de interceptación-, equipadas con un sistema de aviónica confiables que permita una excelente relación humano-máquina. Dejando a un lado la capacidad aire-aire compuesta por misiles y Radares de Barrido Electrónico Activo (AESA, por sus siglas en inglés), la característica más importante para la quinta generación es la baja observabilidad ante radares y buscadores térmicos, lo que es crucial y diferencial respecto a otras aeronaves (James \& Gouré, 2019).

Hoy en día existen investigaciones centradas en el diseño de aeronaves de quinta generación con el propósito de cumplir las necesidades de defensa en el futuro, estas proponen aviones polivalentes y aeronaves tripuladas (Liangliang et al., 2016), en este último caso, los diseños están enfocados en la superioridad aérea (Atique et al., 2016). También se ha combinado la quinta generación con aeronaves no tripuladas UCAV, estas investigaciones incluyen conceptos como el de la inteligencia artificial para así eliminar cualquier dependencia de un piloto (Kong et al., 2020). Adicionalmente, existen propuestas de diseño para aeronaves no tripuladas de quinta generación con la función de caza bombardero (Sepúlveda \& Smith, 2019a), e inclusive, se han caracterizado en detalle misiones donde los UCAv de quinta generación son destinados a misiones de bombardeo de precisión (Sepúlveda \& Smith, 2019b).

Ahora bien, las nuevas exigencias en el área de operaciones de las últimas décadas han sido causantes de la salida de servicio de aeronaves como el Su-25 o del uso de aeronaves más livianas como el A-29B Super Tucano, estas traen consigo una visión futura sobre la adquisición de unidades polivalentes, y a la vez, supone la eliminación de los conceptos especializados dentro de esta industria. Aunque la historia ha demostrado que aeronaves con múltiples versiones del A-10 tienen una alta efectividad en sus misiones, es inevitable el debate sobre la salida de servicio de estos vehículos (Ho, 2018). La principal amenaza para esta aeronave son las reducidas posibilidades de cambios en sistemas de aviónica, proliferación de aeronaves polivalentes, conceptos de furtividad y capacidades de ejecución de múltiples tipos de misiones que suelen ser parámetros de difícil acceso a una aeronave ya manufacturada y antigua (Green \& Zeckhauser, 2019). En retrospectiva, no se sugiere una eliminación de flota, más bien, se exige un camino de transformación y adaptación a los nuevos conflictos que presenta el siglo XxI (Nordhagen, 2018).

Finalmente, este documento desarrolla un proceso de diseño caracterizado por una visión mejorada en las misiones de apoyo aéreo cercano, también denominadas CAS; en dicho proceso se sugiere el uso de aeronaves de combate no tripuladas de quinta generación que incluyen adaptaciones a diferentes velocidades de operación, teniendo en cuenta factores como la precisión, la maniobrabilidad, el uso de la GAU-8/A y los entornos de combate que puedan presentarse en el futuro.

\section{Materiales y métodos}

\section{Requerimientos de diseño}

El diseño de aeronaves de nueva generación para el cumplimiento de misiones tipo CAS se especializa en requerimientos estipulados por aviones de categorías similares (A-10 Thunderbolt II y Sukhoi-25). Además, las exigencias militares que se presentan en la actualidad incluyen características de quinta generación. Por esto, teniendo en cuenta los referentes planteados, se extrajeron los conceptos principales para crear un diseño optimizado, furtivo y con supremacía respecto a sus antecesores. La información se presenta en la tabla 1.

Dentro de las condiciones presentadas en la tabla 1 se contemplan parámetros de supervivencia y de operación, así mismo, se establecen condiciones de diseño enfocadas en la furtividad y aerodinámica de la aeronave. Por otra parte, cada uno de estos requerimientos se debe relacionar directamente con un perfil de misión que permita considerar las facultades y las condiciones que debe contener el diseño para el cumplimiento de sus objetivos como uCAv, por lo cual, en la figura 1 se especifican cada una de las etapas de vuelo para el cumplimiento de misiones tipo cas. 
Tabla 1

Conceptos base para el diseño de una aeronave con objetivos de misión para el apoyo aéreo cercano

\begin{tabular}{c|l|c|l}
$\mathbf{n}^{\circ}$ & \multicolumn{1}{|c|}{ Concepto } & $\mathbf{n}$ Concepto $^{\circ}$ & \multicolumn{1}{|c}{} \\
\hline 1 & Aeronave de ala fija & 6 & $\begin{array}{l}\text { Capacidad de resistencia frente a fuerzas G que se encuentran en los } \\
\text { rangos desde }-3 \text { hasta 7 teniendo en cuenta un máximo peso de despegue. }\end{array}$ \\
\hline 2 & Remotamente piloteado & 7 & $\begin{array}{l}\text { No superar las velocidades de operación de 120 kt, 300 kt y } 900 \text { kt para Stall } \\
\text { Speed, Corner Speed y Maximum Level Speed respectivamente. }\end{array}$ \\
\hline 3 & Baja detectabilidad (RCS<0,005 $\mathrm{m}^{2}$ en configuración limpia) & 8 & $\begin{array}{l}\text { Techo de operación no menor a 40.000 ft y ratio de ascenso mínimo } \\
\text { de 10.000 ft/min. }\end{array}$ \\
\hline 4 & Diseño desarrollado para la supervivencia en combate & 9 & Definición de puntos externos e internos de disposición en carga paga. \\
\hline 5 & GAU-8 Avenger como arma principal & 10 & $\begin{array}{l}\text { Motores que operen bajo las condiciones del ambiente, mediante el uso } \\
\text { del aire como medio de combustión (oxígeno). }\end{array}$ \\
\hline
\end{tabular}

Fuente: elaboración propia

\begin{abstract}
Diseño UCAV - Perfil de misión de combate
Apoyo aéreo cercano

Nivel del mar y cero viento
\end{abstract}

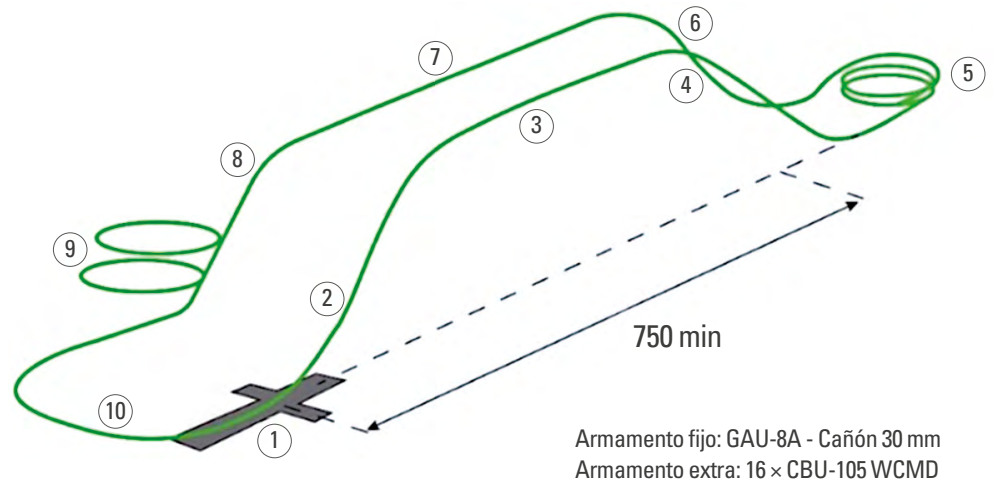

(1) Máximo performance Despegue al nivel del mar

(2) Máximo potencial de ascenso Altitud óptima

(3) Crucero - $750 \mathrm{~min}$ Velocidad/Altitud óptima

(4) Loiter - $120 \mathrm{~min}$ $5.000 \mathrm{ft}$

(5) Combate - $20 \mathrm{~min}$ Corner Speed

(6) Máxima potencia de ascenso Altitud óptima

(7) Crucero retorno $-750 \mathrm{~min}$ Velocidad/Altitud óptima

(8) Descenso al nivel del mar Configuración empuje - Idle

(9) Loiter $-20 \mathrm{~min}$ Autonomía velocidad/Nivel del mar

(10) Aterrizaje 30 min de reserva - Combustible

Figura 1. Perfil de misión de combate relacionada con el apoyo aéreo cercano Fuente: elaboración propia.

La distribución de una misión tipo CAs ha sido clasificada en 10 etapas, como se observa en la figura 1 , lo que permite la inclusión de rangos, tiempos y condiciones principales de operación. Además, en el diseño se puede tener en cuenta la necesidad de una carga paga, determinada por un armamento fijo (GAU-8A-Cañón de $30 \mathrm{~mm}$ ) y un armamento extra ( 16 x CBU-105 y dispensador de munición con corrección de viento), para una posible etapa de combate que se encuentra en la fase 5 de vuelo. Finalmente, con estas consideraciones se procedió a establecer una metodología de diseño que permitiera el desarrollo de un ucav de quinta generación especializado en las misiones de apoyo aéreo cercano.

\section{Metodología de diseño}

El diseño conceptual de la aeronave fue ejecutado mediante de tres etapas que estudiaron acercamiento, diseño y evaluación de cada uno de los procesos con los 
que se obtendrá el uCAv de quinta generación. De igual manera, cada una de estas fases se realizó bajo un flujo metodológico como se muestra en la figura 2, el cual permite obtener un desarrollo lógico y de constante retroalimentación que beneficiaría los resultados finales de la investigación.

Tomando en cuenta la información de la figura 2 se determinaron tres ítems para el desarrollo del diseño: etapas, acciones y herramientas. Así mismo, contienen tres etapas de ejecución denominadas: preanálisis del diseño, diseño de la aeronave y conclusión del diseño. Primero, se realizó una conceptualización general sobre aeronaves que se aplican en misiones tipo CAS, y se evaluaron requerimientos, competencias y bases de datos relevantes. Posteriormente, se procedió con el foco de diseño conceptual de la aeronave incluyendo teorías de diseño, características de aeronaves de quinta generación e información de la etapa anterior, para concluir con la evaluación de los resultados y su aporte para las futuras generaciones de aeronaves. A continuación, se describe cada una de estas etapas.

\section{Etapa 1: preanálisis del diseño}

Para dar inicio al diseño del UCAV, se tuvieron en cuenta cada una de las especificaciones mencionadas en la tabla 1 y los parámetros mostrados en la figura 1. A su vez, se identificaron las principales limitantes que influyen en factores como la capacidad de carga, velocidades y condiciones de operación para el cumplimiento de misiones relacionadas con el apoyo aéreo cercano. Las variables determinadas en el primer acercamiento pautaron los criterios necesarios para la incorporación de una base de datos enlazada

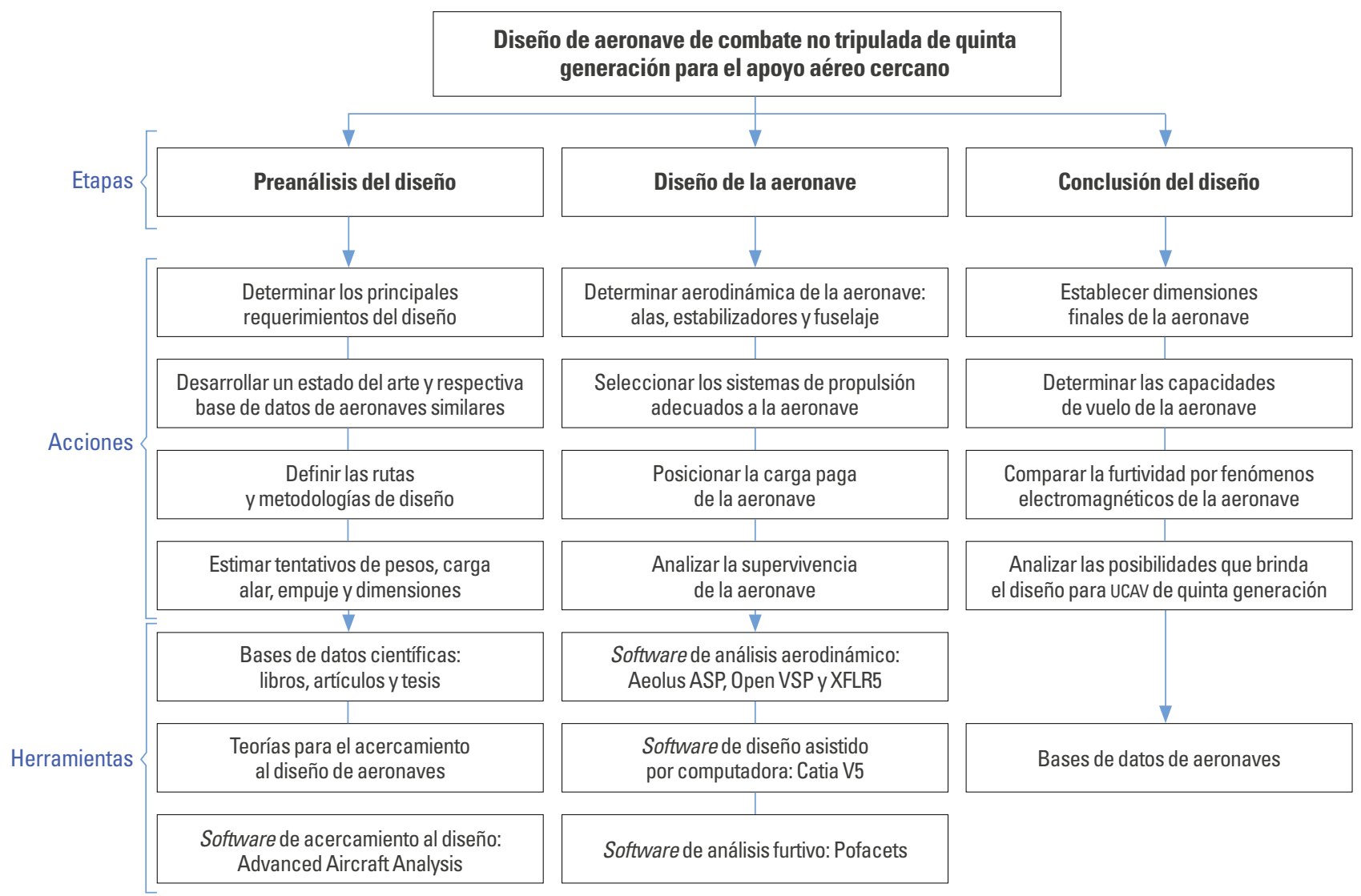

Figura 2. Flujo metodológico para el diseño de un UCAV de quinta generación Fuente: elaboración propia. 
con aeronaves que cumplan misiones cAs, en la que se incluyó información dimensional, de pesos, facultades de operación y otros elementos relevantes para el diseño.

De igual manera, con la base de datos fundamentada en herramientas científicas, como lo estipula la figura 2 , se realizó una estimación de pesos evaluada a través de tres métodos comparativos, los cuales interactuaron entre ellos para la determinación de errores y comprobación de los pesos de diseño -máximo peso de despegue y peso vacío-. Dentro de estos procedimientos se encuentra el uso del software académico Advanced Aircraft Analysis, el cual combina criterios estadísticos para realizar aproximaciones dimensionales. Posteriormente, se plantearon las teorías de Raymer (2018) enfocadas en el desarrollo de cada una de las etapas de vuelo de la misión y, por último, se hizo un acercamiento por medio de fracciones másicas que relacionaron variables aerodinámicas como la eficiencia aerodinámica -razón entre la sustentación y el arrastre- y los factores de consumo de combustible.

Para concluir la etapa de preanálisis, se tomaron en cuenta los pesos obtenidos y requerimientos de operación de la aeronave para determinar los componentes de carga alar (W/S) y la razón entre empuje y peso (T/W). Con la inclusión de variables de operabilidad y aerodinámica se realizó la graficación de un matching plot que considera el cumplimiento de criterios de performance para la selección de un punto de diseño. Además, este diagrama brinda información relacionada con valores de superficie alar y empuje requerido en la aeronave (Raymer, 2018). Dentro de los parámetros de performance se pueden definir restricciones por corner speed, velocidades de operación, distancias de despegue y aterrizaje, maniobrabilidad y condiciones de ascenso.

\section{Etapa 2: diseño de la aeronave}

Después de hacer un acercamiento al diseño definido se dio inicio a la etapa 2; con el apoyo de varios software especializados se realizaron análisis, optimización y verificación de las principales teorías aplicadas al diseño de aeronaves, así mismo, se hizo énfasis en los ítems de aerodinámica y furtividad como se muestra a continuación.

\section{Estimación aerodinámica}

Para la determinación de las superficies aerodinámicas se tuvo en cuenta la fase crucero, ya que es la sección de vuelo más crítica de mayores tiempos y rangos, además, tomando en cuenta los criterios de máximo peso de despegue, condiciones, altitud y velocidades se procede a determinar las características aerodinámicas que debe cumplir el perfil alar. Con estas variables se llevó a cabo una recolección de perfiles que cumplieran estos requerimientos para, luego, evaluarlos en parámetros de coeficiente de sustentación, coeficiente de arrastre, coeficiente de momentos y geometría, este incluye facilidad de manufactura y espesores.

De este modo, el perfil aerodinámico seleccionado, las condiciones de vuelo y la superficie alar -extraída del matching plot- se determinó inicialmente de forma en planta del ala, la cual fue evaluada en el software OpenVSP para obtener las principales variables aerodinámicas - coeficientes de sustentación, arrastre y momentos - y comprobar el cumplimiento de cargas mínimas de sustentación. Así mismo, este primer diseño del ala fue sometido a una optimización geométrica en la que se usó el software Aeolus ASP, por medio de la inclusión de rangos dimensionales - tamaño de cuerda, aflechamientos y envergadura- se buscó la configuración con mayor eficiencia aerodinámica - mayor coeficiente sustentación y menor coeficiente de arrastre-.

Por otra parte, las consideraciones anteriores fueron las bases principales para el diseño de los estabilizadores de la aeronave, los cuales se complementaron con las definiciones generales de la estabilidad dinámica y estática, tanto longitudinal como lateral, demostradas en la ecuación 1 (Bravo-Mosquera et al., 2018).

$C_{M \infty}=a_{w b}\left(\bar{x}_{C G}-\bar{x}_{a c, w b}\right)-a_{t}\left(1-\frac{\partial \varepsilon}{\partial \alpha}\right) \eta_{t} \frac{S_{t}}{S}\left(\bar{x}_{a c, t}-\bar{x}_{C G}\right)$ 
De igual forma, los conceptos de estabilidad se completaron con componentes dimensionales establecidos por comportamientos regulares en aeronaves de categorías similares, los cuales se estipularon por medio de la ecuación 2, ecuación 3 y ecuación 4 (Cidrás-Estévez, 2019).

$$
\begin{gathered}
S H t=C H t \frac{S w * C m a w}{L H t} \\
C H t=\frac{C m w}{C L H t} \\
S V t=C V t * \frac{b w * S w}{L V t}
\end{gathered}
$$

\section{Estimación de furtividad}

El análisis de RCs hace parte de una evaluación que considera los aspectos de geometría y materiales; en este caso, mediante un análisis computacional se podrán ejecutar aproximaciones certeras a la firma de radar que el diseño planteado genera (Chen et al., 2015).

Por otro lado, las simulaciones se realizaron por medio del software Pofacets, un código desarrollado por el Dr. David C., director de facultad del Laboratorio de Antenas y Microondas del Departamento de Ingeniería Eléctrica e Informática de la Escuela Naval de Posgrados en Monterey-California (Jenn, 2019). Con el objetivo de garantizar una cercanía en los resultados del software, se estipularon ecuaciones base de geometrías investigadas, estas se representaron en las ecuaciones de la tabla 2. Así mismo, se realizaron aproximaciones a aeronaves actuales que brindaran un criterio más justificable de tipo aeronáutico.

\begin{tabular}{|c|c|c|c|}
\hline Objeto & Dirección & $\operatorname{RCS}[\sigma]$ & Simbología \\
\hline Esfera & Cualquiera & $\pi r^{2}$ & \multirow{4}{*}{$\begin{array}{l}\text { r: radio } \\
\theta: \text { semiángulo de cono } \\
\text { a: radio de curvatura } \\
\quad \text { del vértice } \\
\mathrm{L}: \text { longitud } \\
\Lambda \text { : longitud de onda }\end{array}$} \\
\hline Cono & Axial & $\frac{\lambda^{2}}{16 \pi} \tan ^{4} \theta$ & \\
\hline Paraboloide & Axial & $\pi a^{2}$ & \\
\hline Cilindro & $\begin{array}{l}\text { Normal } \\
\text { al eje }\end{array}$ & $\frac{2 \pi L^{2}}{\lambda}$ & \\
\hline
\end{tabular}

Tabla 2

Fórmulas aproximadas de RCS para algunos objetos

Fuente: Chen et al. (2005).
Del mismo modo, los conocimientos de RCs en la aeronave brindaron información sobre supervivencia o detectabilidad relativa de esta ante radares militares de diferentes frecuencias y potencias, su modelo matemático está definido bajo las interacciones que existen entre el radar - antena- y el objeto, estipuladas por la ecuación 5 , y la relación del RCS entre la distancia del emisor, la energía incidente y la energía dispersada, representadas por la ecuación 6 (Chatzigeorgiadis, 2004).

$$
R \max =\sqrt{\frac{P t G^{2} \lambda^{2} \sigma}{(4 \pi)^{3} P_{\min }}}
$$

$$
\sigma=\lim _{R \rightarrow \infty} 4 \pi R^{2} \frac{\left|E_{s}^{2}\right|}{\left|E_{i}^{2}\right|}
$$

Para la ecuación 5, Rmax es el máximo rango de detección, $\mathrm{P}_{\mathrm{t}}$ es el poder de transmisión que se refiere a la potencia de microondas en vatios, $\mathrm{G}$ es la ganancia de la antena, de modo que representa la cantidad de energía trasmitida o recibida desde una dirección determinada, $\lambda$ es la longitud de onda, $\sigma$ el RCs del objeto y $P_{\min }$ es la señal mínima detectable. Para la ecuación $6, \mathrm{R}$ es la distancia a la que está el emisor de la onda electromagnética y $\vec{E}_{s}$ junto con $\vec{E}_{t}$ hacen referencia a las amplitudes del campo eléctrico del campo incidente y disperso respectivamente.

\section{Etapa 3: conclusión del diseño}

Finalmente, para concluir el diseño se realizó una verificación de todos los requerimientos definidos en la etapa 1 , además, se compararon las bases de datos obtenidas durante el desarrollo de la investigación y se garantizó los beneficios de la nueva aeronave respecto a la competencia internacional. De igual manera, se demostraron las capacidades que se obtienen al utilizar una aeronave tipo UCAV en el cumplimiento de misiones CAS, teniendo en cuenta las características de las aeronaves de quinta generación. 


\section{Resultados y discusión}

\section{Estimación de características generales de la aeronave}

A través de los procedimientos mencionados en la sección "Etapa 1: preanálisis del diseño" se estableció como componente de mayor relevancia el concepto de las fracciones másicas, con lo cual se logró obtener un acercamiento en los valores de peso de la aeronave que pueden ser demostrados en la tabla 3; estos consideran cada uno de los limitantes relacionados con la misión de apoyo aéreo cercano que ejecutará la aeronave en operación y con los pesos promedios obtenidos al dar uso de las bases de datos.

Tabla 3

Estimación inicial de pesos en la aeronave para la ejecución de misiones tipo CAS

\begin{tabular}{l|c}
\multicolumn{1}{c|}{ Concepto } & Misión 1 \\
\hline Radio [nmi] & 750 \\
\hline Peso carga paga [lb] & 11.240 \\
\hline Peso de despegue [lb] & 61.817 \\
\hline Peso de combustible [lb] & 19.861 \\
\hline Peso vacío [lb] & 30.715 \\
\hline
\end{tabular}

Fuente: elaboración propia.

Los resultados obtenidos en la tabla 3 dan un punto de partida para el diseño de la aeronave, en los que se incluyen los valores de máximo peso de despegue -condición fundamental para el diseño aerodinámico-, peso vacío que permite analizar el peso disponible en la aeronave para la inclusión de sistemas eléctricos, aviónica, motores, entre otros y, finalmente, los componentes de peso de combustible y carga paga que se distribuirán en el volumen de la aeronave para control de centro de gravedad, rangos de operación y tipo de misión a cumplir. Por otro lado, para verificar la certeza de los valores obtenidos por medio de fracciones másicas se llevó a cabo una comparación respecto a los otros métodos de obtención de pesos, como se observa en la tabla 4 , y se realizó el cálculo del error relativo porcentual que se tiene en cada uno de los resultados.

La verificación realizada en la tabla 4 establece porcentajes de error relativo porcentual bajo y totalmente adecuado para la aplicación de estos valores como objeto inicial de diseño. Por ese motivo, al tomar los datos de referencia que incluyen condiciones de vuelo en aterrizaje, despegue y crucero, y al incluir los pesos de despegue y posibles valores aerodinámicos requeridos fue posible realizar la estimación de matching plot para la selección de dimensiones de superficie alar y empuje, como se muestra en la figura 3.

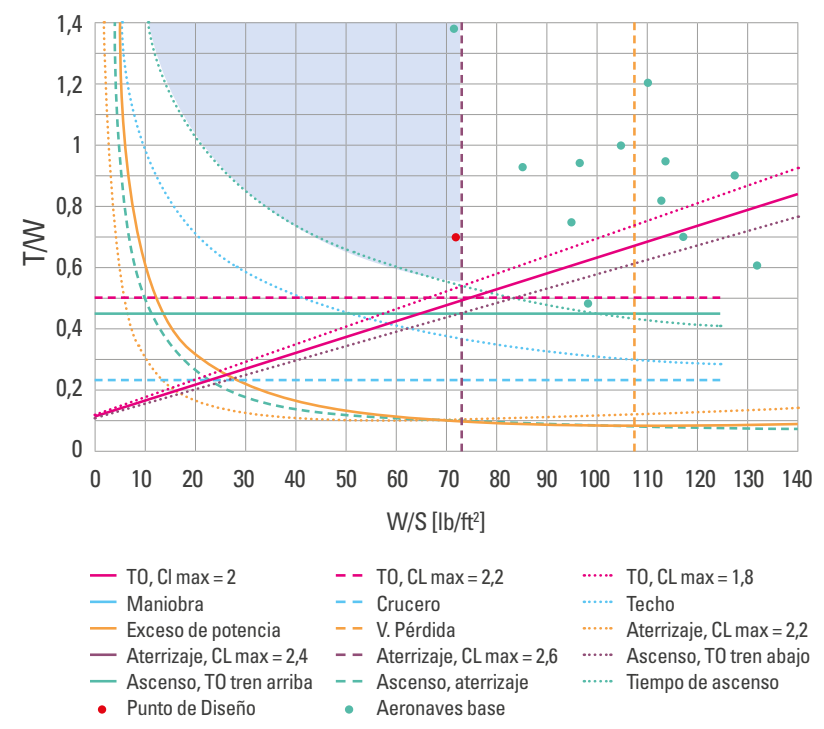

Figura 3. Estimación del punto principal de diseño para la extracción de superficie alar y empuje requerido

Fuente: elaboración propia.

Tabla 4

Verificación de los resultados obtenidos por fracciones másicas

\begin{tabular}{l|c|c|c|c|c|c|c}
\multicolumn{1}{c|}{ Parámetro } & Fracciones másicas & Base de datos & Error (\%) & Aaa software & Error (\%) & Consumo combustible & Error (\%) \\
\hline Peso de despegue [lb] & 61.817 & 61.817 & 0 & 61.861 & 0,07 & 61.817 \\
\hline Peso vacío [lb] & 30.715 & 32.613 & 6,17 & 30.161 & 1,8 & 0 \\
\hline
\end{tabular}

Fuente: elaboración propia. 
Las relaciones obtenidas para la aeronave especifican el cumplimiento de todos los limitantes planteados en la sección "Etapa 1: Preanálisis del diseño" que además, relaciona la inclusión de estándares de diseño como lo es el bajo consumo de combustible y la baja necesidad de un área alar con amplias dimensiones. Estos resultados son significativos respecto a un área alar igual a $80 \mathrm{~m}^{2}$ y un empuje mínimo de $43.271 \mathrm{lbf}$, los cuales son cercanos a aeronaves de altas prestaciones como lo es el Lockheed Martin F-22 Raptor.

Por lo anterior, las bases de empuje requerido implican la preselección de un motor adecuado a la aeronave, mediante una base de datos aplicada a los motores de mayor relevancia en el campo, por lo que se optó por el uso de dos F199-PW-100, caracterizados por un alta eficiencia en el consumo de combustible, capacidad de hasta 35.050 lbf con módulo de afterburner y un peso de $2.998 \mathrm{Lb}$. Adicionalmente, sus referencias en la industria lo hacen un motor de excelentes prestaciones (Deskin \& Yankel, 2002) que se encuentra en constante evolución para las nuevas aeronaves que ingresarán en manufactura en las próximas décadas.

\section{Diseño de superficies aerodinámicas}

De acuerdo con los estándares mencionados en la subsección anterior, los requerimientos de diseño de superficies aerodinámicas se acoplaron al cumplimiento del máximo peso de despegue, maniobras de alta relevancia para las aeronaves de tipo CAS, conceptos de arrastre aerodinámico y otros elementos de importancia en la operación regular. Por ello, el proceso de diseño inició con el desarrollo de una base de datos de perfiles aerodinámicos que cumplieran características de coeficiente de sustentación igual o mayor a 0,3 a un ángulo de ataque de 0 , valores máximos de espesor del $15 \%$ respecto a la cuerda y geometrías de fácil manufactura.

Tomando en cuenta la etapa crucero como la fase de vuelo más crítica se analizaron cada uno de los perfiles en los que se realizó una matriz de selección y comparación que evalúa factores de forma geométrica - facilidad de manufactura e influencia en la generación de traza de radar-, posibilidad de twist aerodinámico -manipulación en espesores y adaptación a los factores limitantes generados por el cono de mach-y finalmente las principales características aerodinámicas: coeficiente de momento, coeficiente de sustentación y coeficiente de arrastre. Las evaluaciones aerodinámicas de cada uno de los perfiles se hicieron por medio del software XFLR5, el cual tiene una validez regular hasta un Reynolds de 3.000 .000 mediante teorías de ala infinita o de perfiles, por lo cual, para esta primera sección de evaluación y comparación es lo suficientemente aceptable, - sin embargo, será más estricta al momento de determinar valores en el ala-. Con lo anterior, se logró obtener como perfil seleccionado el NACA 63-306 con los resultados que se muestran en la figura 4.
Figura 4. Representación general sobre el perfil aerodinámico NACA 63-306 (Evaluación $\mathrm{Re}=3.000 .000$ )

Fuente: elaboración propia.

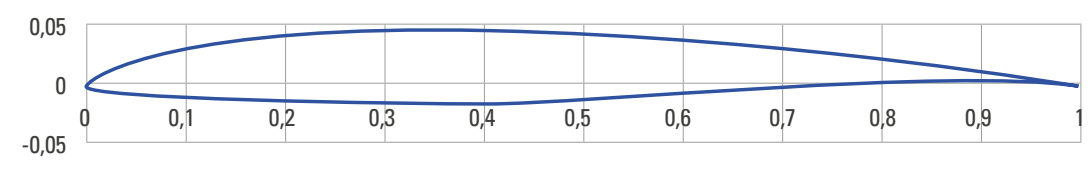

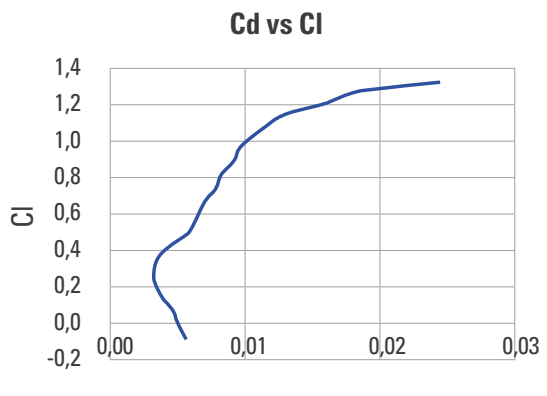

$\mathrm{Cd}$

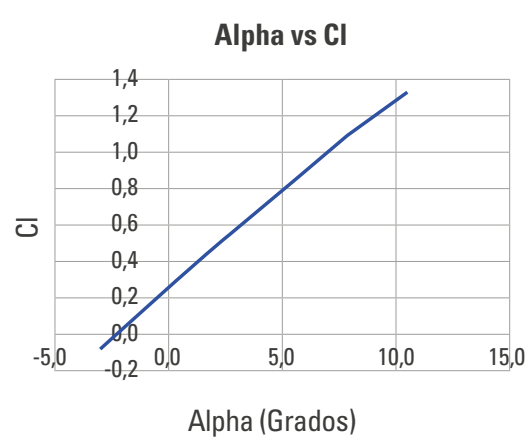


Como se observa en la figura 4 su máximo espesor es relativamente pequeño, lo que permite una escalabilidad superior o menor en los rangos de generación de nuevos perfiles para el twist aerodinámico. A la vez, efectúa cambios en este concepto entre los valores de $2 \%$ y $10 \%$ respecto a la cuerda unitaria del perfil. Además, la composición de la geometría se adapta apropiadamente a la posibilidad de diseño de superficies sustentadoras de forma adiamantada, aplicando esto a las bases principales de furtividad; su curvatura representativa puede ser estudiada para una manufactura eficiente y que no presente problemas en ninguno de los procesos para la obtención del producto. Posteriormente, con los resultados obtenidos en los análisis de requerimientos de máximo peso de despegue, empuje requerido y otros parámetros, se establecieron los limitantes dimensionales que se encuentran en la tabla 5.
Tabla 5

Conceptos dimensionales para el diseño de la superficie de sustentación

\begin{tabular}{l|c|c}
\multicolumn{1}{c|}{ Variable } & Valor & Unidad \\
\hline Superficie alar (S) & 871,8 & $\mathrm{ft}^{2}$ \\
\hline Relación de aspecto (AR) & 3 & $\mathrm{~N} / \mathrm{A}$ \\
\hline Envergadura (b) & 50,5 & $\mathrm{ft}$ \\
\hline
\end{tabular}

Fuente: elaboración propia.

Teniendo en cuenta estos limitantes se definieron diferentes posiciones geométricas con variaciones en aflechamiento, cuerda de raíz y cuerda de punta. Sumado a ello, se acopló un modelo simplificado sobre la primera formación de onda de choque para un mach de 1,2 sobre la superficie de sustentación, aplicando los máximos rangos de velocidad en la operación de la aeronave como se observa en la figura 5.
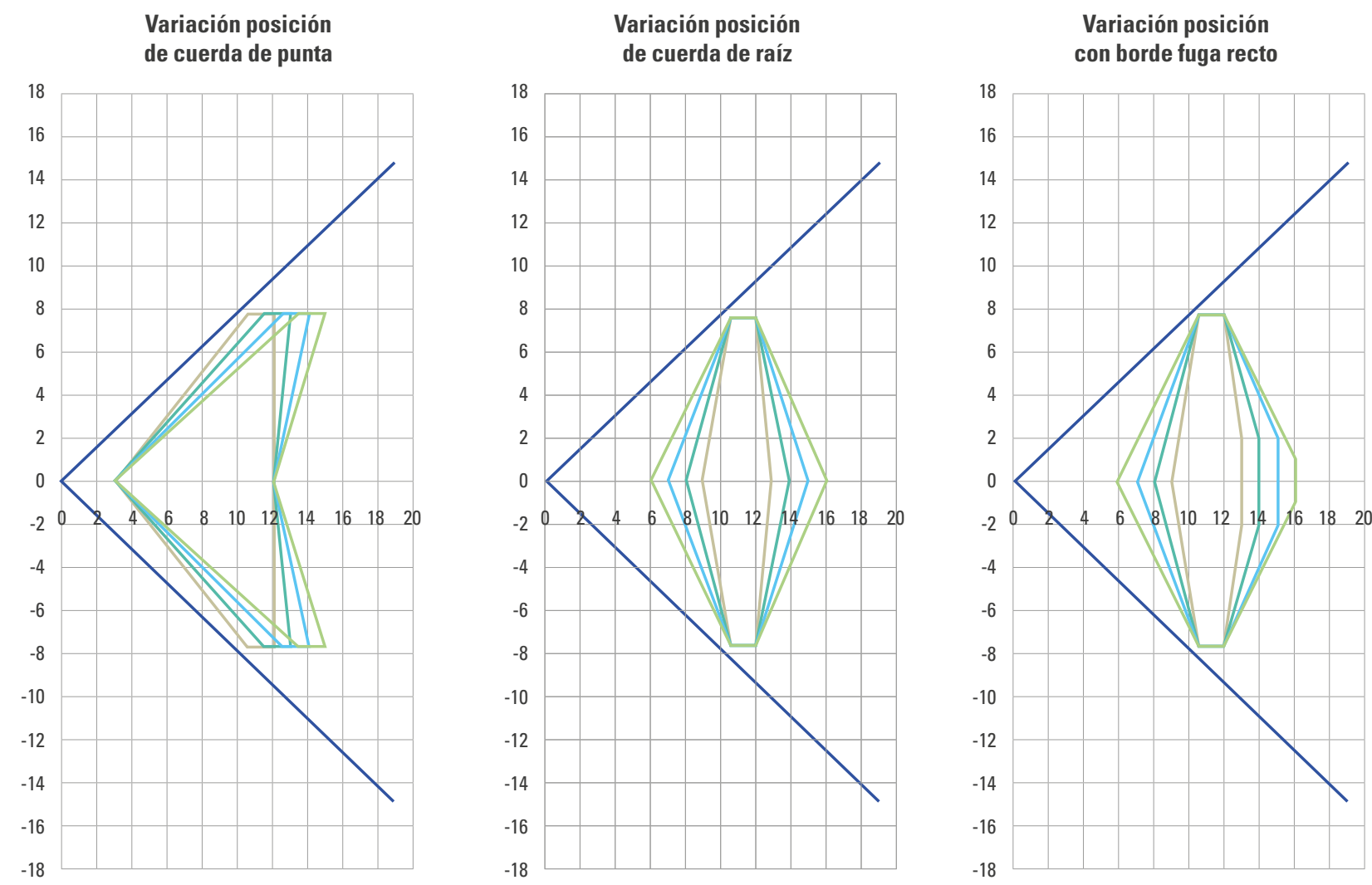

Figura 5. Posibles variaciones en geometría alar en metros, la línea azul representa el modelo de onda de choque a 1,2 mach Fuente: elaboración propia. 
Estas geometrías son acompañadas de un análisis aerodinámico en OpenVSP y un modelo de optimización en Aeolus ASP, como se especificó en la sección "Etapa 3: conclusión del diseño" que permite establecer bajo los criterios de manufactura, aerodinámica y capacidad volumétrica, una forma altamente eficiente con la capacidad de garantizar altos estándares de operabilidad en la aeronave. Además, los conceptos asociados a furtividad incluyen el desarrollo de twist aerodinámico, un proceso en el que por medio de modificaciones en valores de espesor, respecto al perfil principal seleccionado, se obtiene una forma adiamantada en toda la envergadura de la superficie de sustentación, lo cual incluye una disminución en RCS considerable que contempla la conceptualización final observada en la figura 6 .

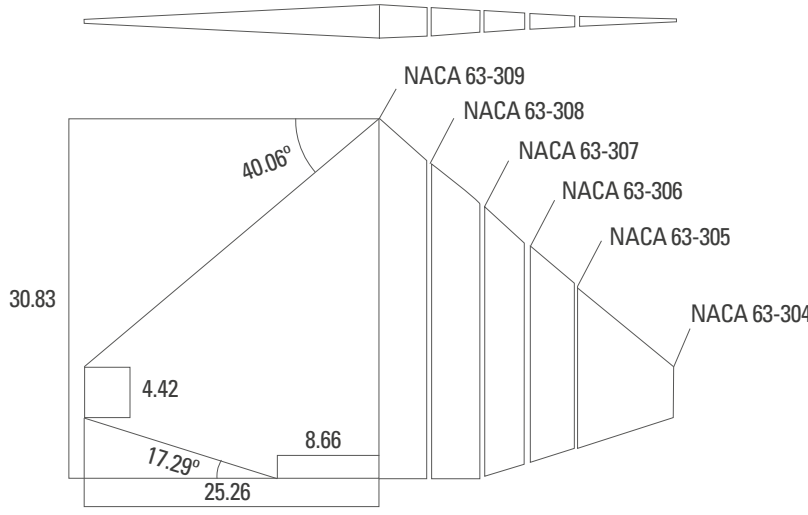

Figura 6. Dimensiones finales de la superficie de sustentación en ft y vista adiamantada por twistaerodinámico

Fuente: elaboración propia.
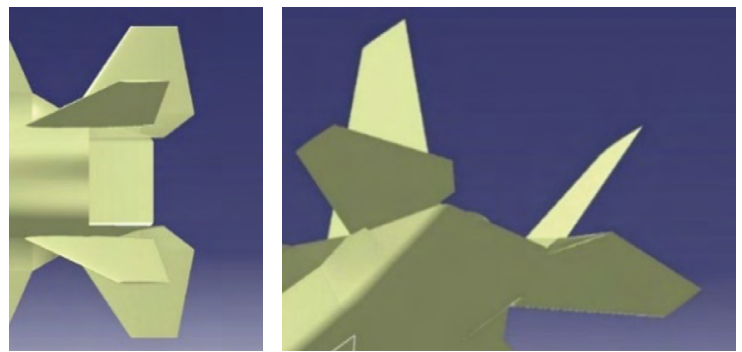

Figura 7. Ilustración sobre los estabilizadores en el fuselaje de la aeronave Fuente: elaboración propia.
Después de verificar la noción final de la superficie de sustentación ilustrada en la figura 6 , se especifica un modelo totalmente adaptado a las necesidades de operabilidad que se presentan para los próximos años, lo que incluye una capacidad volumétrica amplia para beneficios de carga interna, adaptabilidad a parámetros de furtividad por medio de formas adiamantadas, y el cumplimiento de requisitos aerodinámicos generales, como lo son sustentación para máximo peso de despegue y bajo arrastre que garantice altos rangos de vuelo.

Más adelante, como parte del trabajo con los resultados obtenidos para el diseño de las superficies de estabilidad, el cual incluye la adaptación de las ecuaciones 1-4, se estableció un diseño de cola tipo compuesto que contempla un estabilizador horizontal y un estabilizador en $\mathrm{V}$, cuyos parámetros dimensionales se encuentran en la tabla 6. De igual manera, en la figura 7 se observa una posible adaptación de estos elementos en el fuselaje de la aeronave.

Tabla 6

Dimensiones finales de las superficies de estabilidad por semienvergadura

\begin{tabular}{l|c|c|l|c|c}
\multicolumn{3}{c}{ Estabilizador horizontal } & \multicolumn{3}{c}{ Estabilizador en V } \\
\hline \multicolumn{1}{c|}{ Variable } & Valor & Unidad & Variable & Valor & Unidad \\
\hline Superficie & 140 & $\mathrm{ft}^{2}$ & Superficie & 106,5 & $\mathrm{ft}^{2}$ \\
\hline Cuerda raíz & 11,5 & $\mathrm{ft}$ & Cuerda raíz & 12,4 & $\mathrm{ft}$ \\
\hline Cuerda punta & 5,0 & $\mathrm{ft}$ & Cuerda punta & 5,0 & $\mathrm{ft}$ \\
\hline Aflechamiento & 40 & Grados & Aflechamiento & 40 & Grados \\
\hline Semienvergadura & 17,0 & $\mathrm{ft}$ & Semienvergadura & 11,0 & $\mathrm{ft}$ \\
\hline
\end{tabular}

Fuente: elaboración propia.

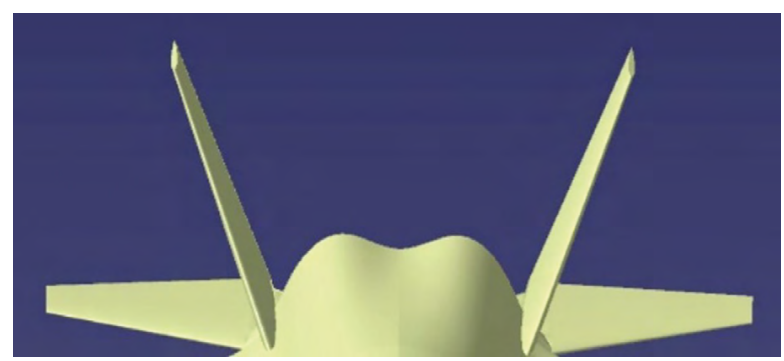


Los valores dimensionales obtenidos para estas superficies y su posicionamiento, son de fácil asimilación con los contextos evaluados en las aeronaves de quinta generación, donde la versatilidad de una cola en configuración compuesta amplifica las variables de maniobrabilidad, control y cambio de aptitud en vuelo. A su vez, estos resultados reflejan ciertas similitudes con aeronaves como el Lockheed Martin F-22 Raptor, lo que garantiza cada uno de los procesos realizados. A lo anterior, se suma que la estabilidad de la aeronave responde a la fase de vuelo crucero y específica a sobremedida la excelente aplicabilidad del diseño sobre la operabilidad misma.

\section{Furtividad y supervivencia}

\section{Verificación pofacets}

Teniendo en cuenta los modelos matemáticos observados en la tabla 2 , se realizó una comparación en el software Pofacets con el objetivo de determinar los valores representativos de RCS. En este caso se tomó un cilindro como objeto de prueba, cuya geometría y resultados se aprecian en la tabla 7.

Tabla 7

Experimento de cilindro en Pofacets

\begin{tabular}{l|c|l|c}
\multicolumn{1}{c|}{ Denominación } & $\begin{array}{c}\text { Valor/ } \\
\text { Referencia }\end{array}$ & \multicolumn{1}{|c|}{ Denominación } & $\begin{array}{c}\text { Valor/ } \\
\text { Referencia }\end{array}$ \\
\hline $\begin{array}{l}\text { Permitividad } \\
\text { Relativa }\end{array}$ & PEC & $\begin{array}{l}\text { RCS promedio } \\
\text { experimental [m²] }\end{array}$ & 0,00261 \\
\hline Radio [m] & 0,063 & $\begin{array}{l}\text { RCS promedio } \\
\text { experimental dBsm }\end{array}$ & 25,12 \\
\hline Altura [m] & 5 & $\begin{array}{l}\text { RCS promedio } \\
\text { experimental [m²] }\end{array}$ & 325,03 \\
\hline Longitud de onda [m] & 0,03 & RCS teórico [m²] & 329,87 \\
\hline $\begin{array}{l}\text { RCS promedio } \\
\text { experimental [dBsm] }\end{array}$ & $-25,84$ & Error [\%] & 1,47 \\
\hline
\end{tabular}

Fuente: elaboración propia.

Con los valores alcanzados en la tabla 7 , se obtuvo un error del $1,47 \%$ con respecto a los resultados dados por las ecuaciones de la tabla 4, lo que determina aceptabilidad entre la teoría y la simulación de
Pofacets. No obstante, la conceptualización en aeronaves involucra combinación de formas y geometrías complejas, las cuales no poseen una ecuación determinada, por este motivo, se planteó el uso del modelo por defecto de la aeronave F-16 teniendo en cuenta las mismas características de la simulación anterior. Los resultados obtenidos se encuentran en la figura 8.

Después de tomar en consideración el análisis de la figura 13 , se especificó que el F-16 obtuvo un RCs promedio de -3,34 dBsm, lo cual es equivalente a una sección transversal de radar de 0,46 $\mathrm{m}^{2}$. Según datos reportados, el RCs de esta aeronave se encuentra normalmente entre rangos de 0,1 y $1,2 \mathrm{~m}^{2}$ de acuerdo con su versión, por lo cual se puede confirmar que el valor obtenido por Pofacets es confiable, puesto que se encuentra en estos valores (Touzopoulos et al., 2018).

\section{D RCS Plot of F16 Model: RED:RCS- $\theta$, green:rcs- $\phi$}

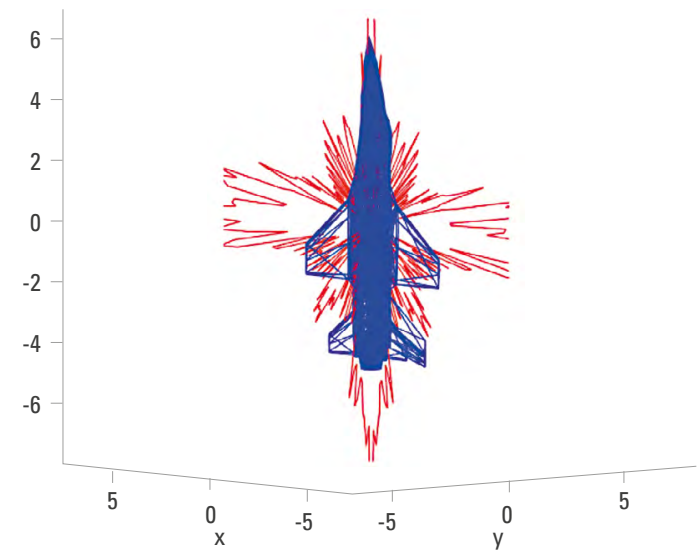

target: F16 solid: $\theta$ dashed: $\phi \quad \phi=0$ wave (m): 0.03

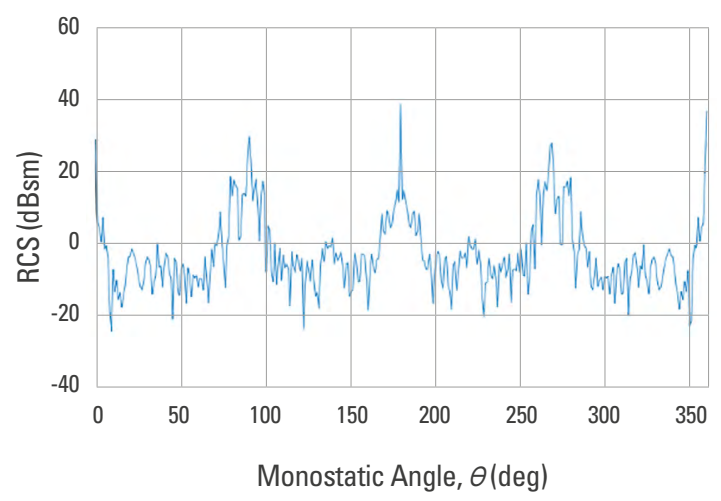

Figura 8. Arriba, Modelo del F-16 por defecto, y abajo, los resultados obtenidos en Pofacets

Fuente: elaboración propia. 


\section{Diseño de fuselaje}

Para dar inicio al diseño del fuselaje se estipularon diferentes geometrías analizadas bajo su Rcs, con el objetivo de encontrar los principales parámetros que guían el modelo furtivo: criterios aerodinámicos, manufactura y capacidad de carga. En la figura 9 se encuentran algunas de las formas consideradas.

Se determina que la forma seleccionada será la hexagonal, debido a que proporciona un mejor acondicionamiento sobre la ubicación de carga paga, aviónica y sistemas internos con la combinación de excelentes resultados en valores de RCS. En otras palabras, este tipo de geometría mantiene un equilibrio entre las variables de furtividad y aerodinámica. En la figura 10 se aprecia el diagrama RCS de la geometría seleccionada. geometría en nariz encargada del almacenamiento de los núcleos de procesamiento, aviónica y otros sistemas de vital importancia, que de igual manera adapta la versatilidad para el posicionamiento de la GAU-8A. Todas estas características se encuentran en la figura 12.

Como se puede observar en la figura 12, el diseño está enfocado en mantener una configuración limpia, lo que permite llevar la carga paga en bahías internas, esto es de gran beneficio para mantener la furtividad de la aeronave SuperCAS. Con esta última conceptualización se realizó un análisis del RCS del fuselaje para determinar un diseño furtivo en este, como se muestra en la figura 13.

En la figura 13 se logró un registro promedio de área detectable con valor de 0,0016 $\mathrm{m}^{2}$ igual a $-27,93 \mathrm{dBsm}$ de señal reflejada. Se observa en esta misma figura la

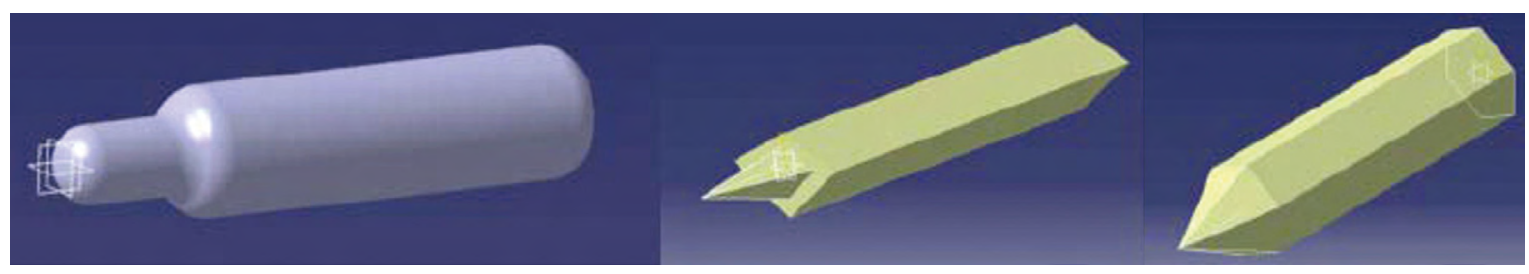

Figura 9. Principales geometrías simuladas en Pofacets para definición de un fuselaje furtivo, de izquierda a derecha cilíndrica, rombo, hexagonal Fuente: elaboración propia.

La figura 10 tiene un valor de señal promedio por debajo de los $-20 \mathrm{dBsm}$ el cual se refiera a un Rcs de $0,01 \mathrm{~m}^{2}$, por lo que se procedió a modificar el diseño con base en esta forma. El fuselaje diseñado consiste en un cuerpo similar a un hexágono, sin embargo, se le acondicionaron aptitudes aerodinámicas que se acoplaran a los requisitos de diseño contemporáneos, por ejemplo, la parte inferior adquiere características más rectangulares para el posicionamiento de bahía de armas, mientras que la zona superior se capacita para la ubicación de la toma de aire y sus respectivos criterios. En la figura 11 se observa el fuselaje obtenido.

Es importante resaltar que los ángulos del fuselaje son cercanos a valores de $60^{\circ}$, para generar una superficie que no devuelva las ondas electromagnéticas directamente al receptor y poder ajustar la forma para el armamento interno. También se incluyó una

\section{RCS in $\mathrm{dBsm}$ for $\mathrm{phi}=\mathbf{0}$ degrees}

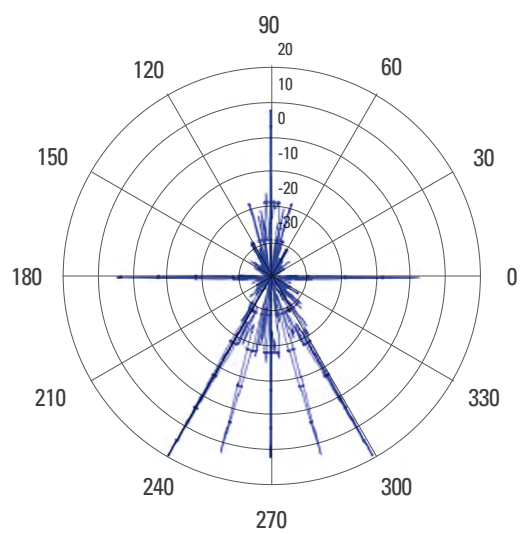

Figura 10. Diagrama RCS en dBsm lograda por el Pofacets, donde $90^{\circ}$ es la nariz de la geometría

Fuente: elaboración propia. 


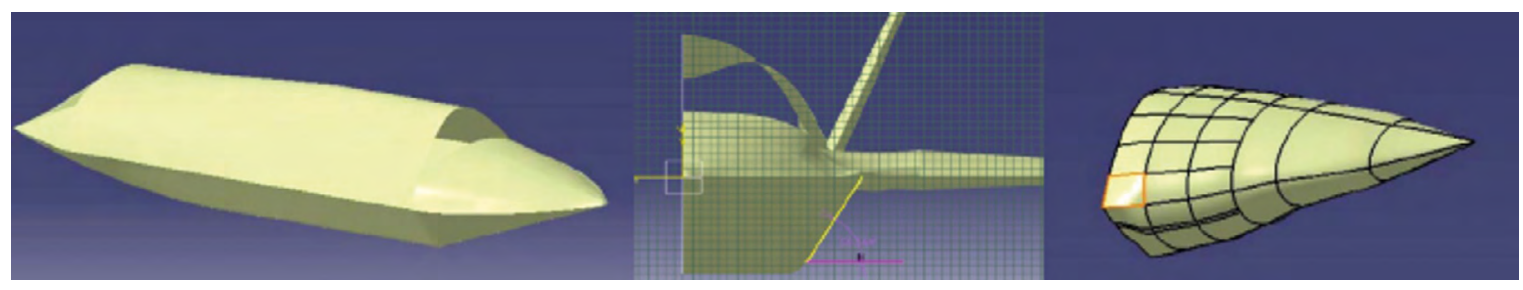

Figura 11. Fuselaje furtivo resultante de los análisis, de izquierda a derecha se presenta: isométrico, vista frontal y radomo Fuente: elaboración propia.

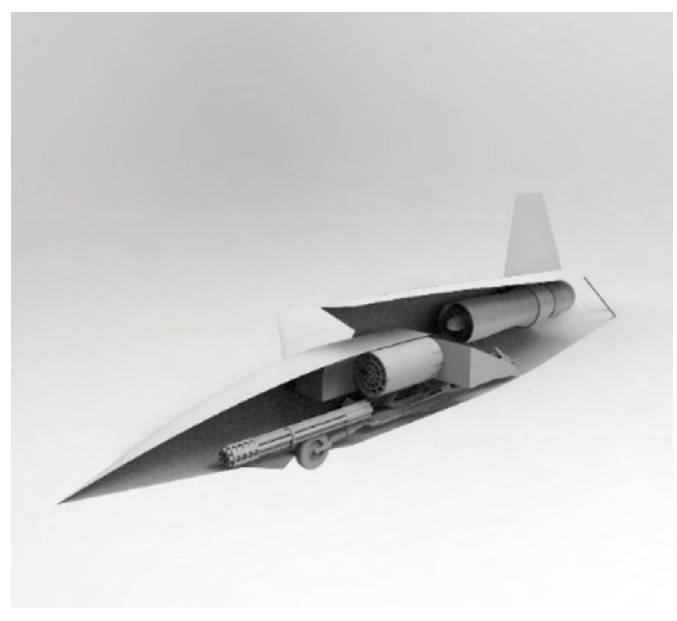

Figura 12. Acercamiento a la vista interna de la aeronave donde se pueden encontrar el motor, GAU-8A, espacios habilitados para aviónica y sistemas internos, entre otros

Fuente: elaboración propia.

existencia de puntos débiles en la parte trasera y delantera de la aeronave $\left(0 / 360^{\circ}\right.$ y $\left.180^{\circ}\right)$, estos pueden ser causados por la simplificación realizada que permite la conceptualización de paredes, donde las ondas electromagnéticas podrían chocar en un ángulo de incidencia de $90^{\circ}$. Este fenómeno mencionado también puede estar presente en las zonas laterales del fuselaje $\left(90^{\circ}\right.$ y $\left.270^{\circ}\right)$, y generar un aumento considerable en la señal emitida. No obstante, el posicionamiento de estos sectores permite tener su ubicación en zonas específicas donde la posibilidad de incidencia de las ondas es muy baja, debido al tipo de operación y velocidades que contiene la aeronave.

Finalmente, se puede concluir que el diseño del fuselaje es exitoso en cuestiones de furtividad, puesto que, en ambos casos, el área transversal y la longitud son de la magnitud de centímetros y centímetros
RCS in $\mathrm{dBsm}$ for $\mathrm{phi}=\mathbf{0}$ degrees

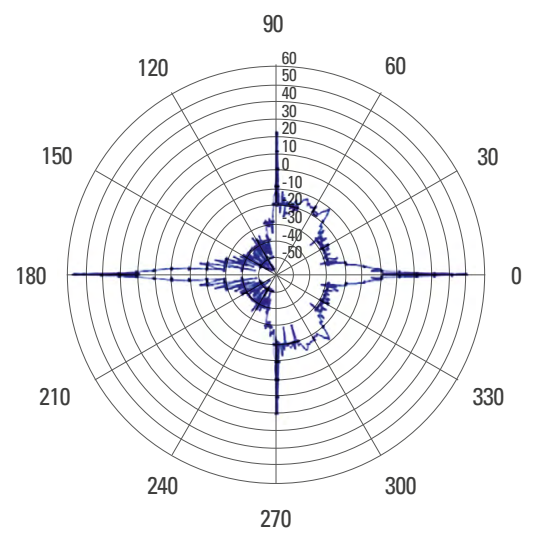

Figura 13. Diagrama RCS del fuselaje de la aeronave donde a $0^{\circ}$ se encuentra la nariz

Fuente: elaboración propia.

cuadrados, en este caso $16 \mathrm{~cm}^{2}$ es menor al pedido en los requerimientos de la tabla 1 que es de $0,005 \mathrm{~m}^{2}$.

\section{Diseño aeronave furtiva}

Con los resultados obtenidos en el fuselaje se construyó un modelo simplificado de la aeronave que permite su análisis en Pofacets, por lo cual, las simplificaciones se encuentran enfocadas en el uso de geometrías sencillas y la eliminación de espacios internos vacíos -sólido completo-. Estos cambios generan un aumento en los valores de RCS debido a la aparición de paredes que no existirían en un modelo real, sin embargo, todas estas consideraciones se tomaron en cuenta para los resultados finales. A continuación, en la figura 14 se encuentra la representación 3D del modelo completo de la aeronave simplificada. 

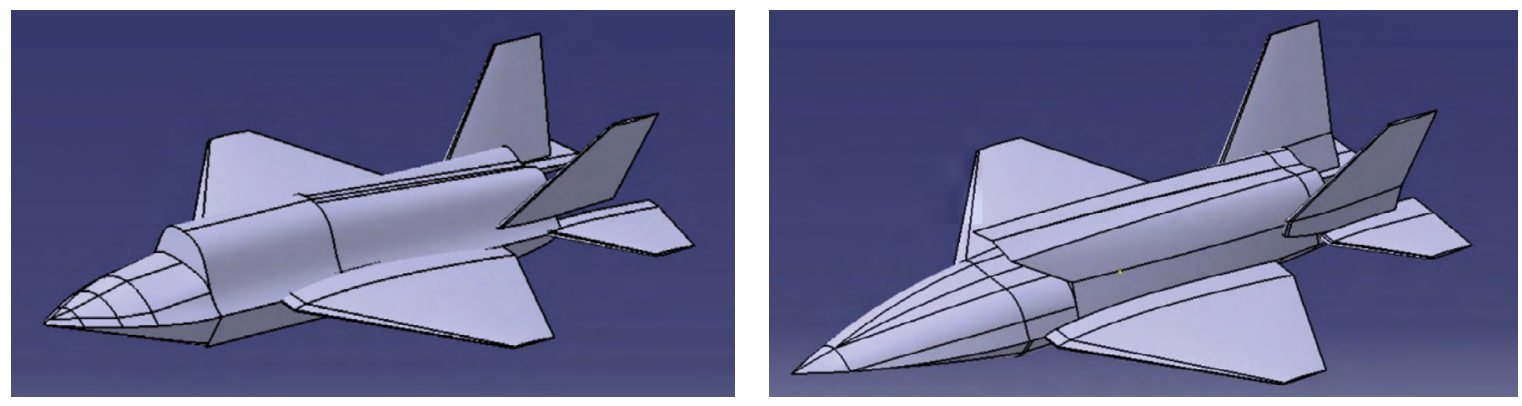

Figura 14. Modelo y evolución de la geometría de la aeronave simplificada Fuente: elaboración propia.

RCS in dBsm for $p h i=0$ degrees

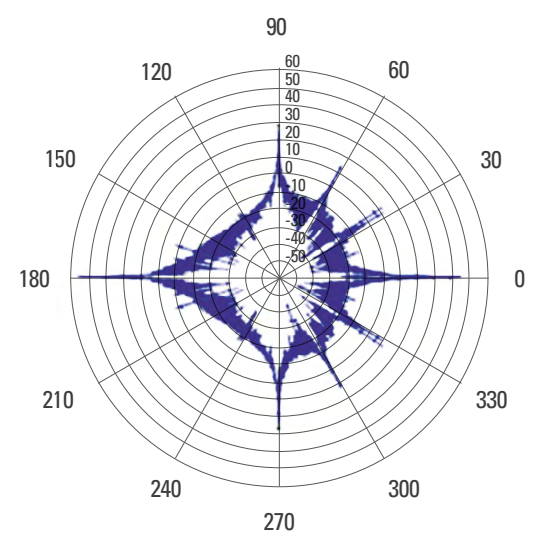

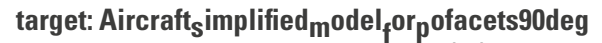
solid: $\theta$ dashed: $\phi \quad \phi=0$ wave $(\mathrm{m}): 0.03$

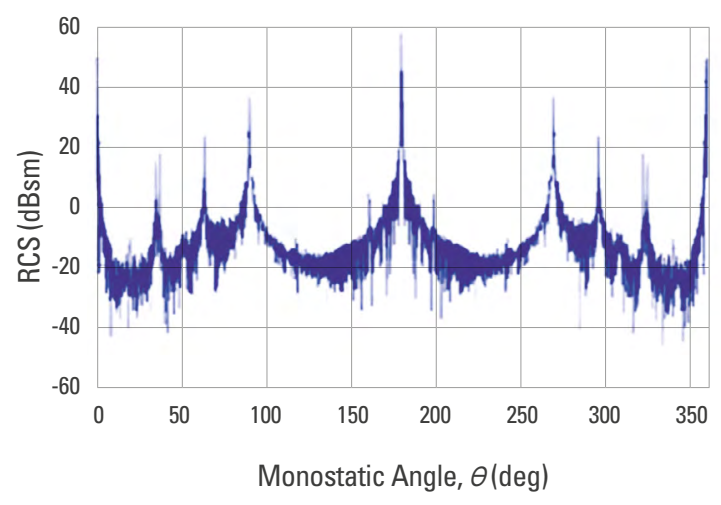

Figura 15. Análisis de RCS usando Pofacets con un paso de ángulo de 0,1. Al lado izquierdo el diagrama polar y al derecho el diagrama rectangular Fuente: elaboración propia.

Las condiciones de simulación involucran la aeronave con una permisividad de PEC - forma ideal-, una frecuencia de $10 \mathrm{GHz}$ y una longitud de onda de $0,03 \mathrm{~m}$, la cual pertenece a banda X -común en aplicaciones militares-. Por último, se planteó un análisis monoestático -emisor y receptor de la señal en un mismo punto- bajo un posicionamiento de $360^{\circ}$ alrededor de la aeronave, los resultados obtenidos se encuentran en la figura 15.

A primera vista, el modelo de la aeronave simplificado de la figura 15 , ubica en sus resultados picos en los ángulos $90^{\circ}, 180^{\circ}, 270^{\circ}$ y $360^{\circ}$, pertenecientes a las zonas alteradas geométricamente por la simplificación, estos son interpretados como un fenómeno propio del mismo proceso. Por otra parte, el RCS promedio final es de $-14,18 \mathrm{dBsm}$ y hace referencia a un área de 0,038 $\mathrm{m}^{2}$; este valor puede ser comparado con aeronaves furtivas de gran reconocimiento como el B-2 Spirit (Air Force Magazine, 2019), donde se obtienen unos valores menores a esta. De igual forma el área obtenida es proporcional a la identificación de un ser humano por medio de un radar.

Aun así, tomando en cuenta la investigación realizada por el Departamento de Ciencias de la Academia de la Fuerza Aérea Helénica, mediante el software Pofacets se estima que la inclusión de Material Absorbente de Radiación (RAM, por sus siglas en inglés) genera una disminución de $10 \mathrm{dBsm}$ respecto a un análisis únicamente geométrico (Touzopoulos et al., 2018), por lo tanto, se logra concluir que la señal promedio obtiene un valor final de $-24,18 \mathrm{dBsm}$ para un RCS de $0,0038 \mathrm{~m}^{2}$. Con este valor final de Rcs se determina que la aeronave diseñada se encuentra en los rangos representativos para la $5 .^{a}$ generación, con un acercamiento 
a las condiciones del F-35 (-21 dBsm para 0,0079 m²) lo que permite superar aeronaves de $4 .{ }^{a}$ generación como el F-16 (Touzopoulos et al., 2018).

\section{Detectabilidad frente a radares}

Al tomar como referente la ecuación 5 se puede realizar procesos matemáticos que permitan determinar el rango de detección de un objeto respecto al RCS de este, para definirlo bajo la ecuación 9 (Wolff).

Rango de detección $=\frac{\text { Rango de detección del radar }}{\sqrt[4]{\frac{R C S \text { mínimo del radar }}{\text { Rcs del objetivo }}}}$

Así mismo, al partir de un ambiente de evaluación que simula la posibilidad de encuentro con amenazas de aeronaves como los Flanker (Aeronaves Sukhoi del 27 al 35) y Fulcrum (Mig-29 y Mig-35) en todas sus versiones se logra efectuar un listado de los principales radares utilizados por este tipo de tecnologías, además, se incluye el radar estadounidense APG 71, común en aeronaves como el F-14. Con esta información y de acuerdo con la relación estipulada en la ecuación 9 se hicieron aproximaciones de rango de detectabilidad del diseño propuesto en condiciones ideales para máximo rendimiento; los resultados se encuentran en la tabla 8 y han sido ilustrados en la figura 16.

Tabla 8

Rangos de detección de la aeronave respecto a los diferentes radares

\begin{tabular}{l|c|c}
\multicolumn{1}{|c|}{ Radar } & $\begin{array}{c}\text { Rango de detección del } \\
\text { objeto de RCS min para } \\
\text { un objeto de } \mathbf{1} \mathbf{~ m}^{2} \text { [nmi] }\end{array}$ & $\begin{array}{c}\text { Rango de detección } \\
\text { de la aeronave [nmi] }\end{array}$ \\
\hline N001 Myech & 47,5 & 11,8 \\
\hline N001VE & 76,1 & 19,0 \\
\hline N001VEP & 76,1 & 19,0 \\
\hline N001VE-Pero & 102,0 & 25,3 \\
\hline N011 & 75,0 & 18,64 \\
\hline N011M BARSV1 & 89,5 & 22,2 \\
\hline N011M BARSV2 & 117,5 & 29,2 \\
\hline Irbis-E & 153,0 & 38,0 \\
\hline APG 71 & 256,4 & 63,7 \\
\hline
\end{tabular}

Fuente: Kopp et al. (2008).
Detectabilidad de la aeronave ante varios radares

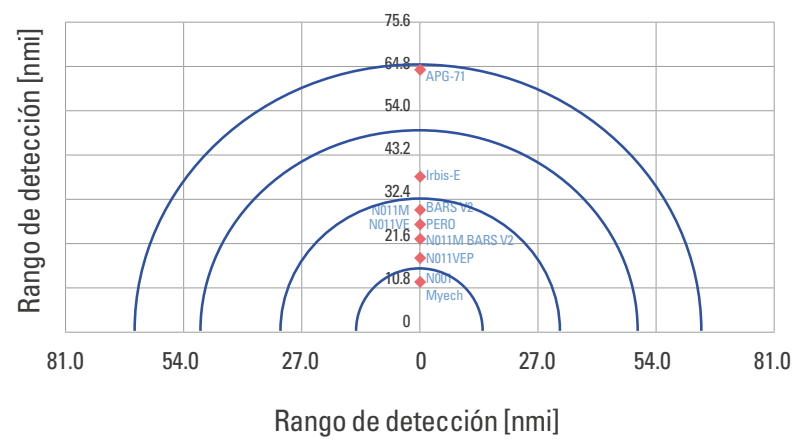

Figura 16. Ilustración de los rangos de detectabilidad de la aeronave frente a los principales radares

Fuente: elaboración propia.

Los resultados obtenidos representan un perímetro de efectividad furtiva de la aeronave frente a posibles amenazas que se involucrarían en su operación regular, un concepto de vital relevancia para la adaptación a las nuevas tecnologías que presentan las aeronaves de 5. a generación.

Como se puede observar en la tabla 8 , el $45 \%$ de los radares estudiados requieren estar a menos de 40 $\mathrm{km}$ de distancia de la aeronave para efectuar una detección de esta, además, el 33 \% de los radares requieren estar en un rango entre 40 y $50 \mathrm{~km}$ de distancia para tener una detección efectiva. A partir de estos resultados se puede estimar que la gran parte de los radares presentes en las aeronaves de superioridad aérea rusa no superan la efectividad de $50 \mathrm{~km}$ de distancia. Por otra parte, los dos últimos radares correspondientes al Irbis-E, presentes en aeronaves como el Sukhoi-35 y el APG-71, representan la mayor amenaza con la capacidad de superar los $70 \mathrm{~km}$ de distancia para la detectabilidad del diseño planteado.

No obstante, cabe recalcar que los rangos de misiles aire-aire de larga y media distancia más actuales conservan un rango máximo efectivo menor a $74 \mathrm{~km}$ de distancia. Además, teniendo en cuenta que la detectabilidad de la aeronave se ve representada en primera instancia bajo una señal correspondiente a un objeto de $0,0038 \mathrm{~m}^{2}$, se estipulan tiempos de identificación frente a la aeronave desconocida, lo que beneficia la supervivencia de esta. Finalmente, lograr un 
sistema de supervivencia completa requeriría la incorporación de un sistema AWACS, propio de aeronaves de alerta temprana efectivas contra radares de rangos mayores a $400 \mathrm{~km}$ y con la capacidad de detectar amenazas antes de que la aeronave se vea comprometida.

\section{Diseño final}

El diseño de la aeronave termina en la conjunción de todos los parámetros, cálculos, consideraciones y requerimientos mencionadas a lo largo de este artículo, reflejadas en una combinación entre el ala, fuselaje y estabilizadores. Finalmente, se logra obtener un modelo revolucionario y de fácil adaptación como se observa en la figura 17.

En la figura 17 se muestran las dimensiones principales de la aeronave en sus respectivas vistas e isométrico. Como se puede detallar, esta ucav posee dimensiones similares a las de un F-22 en cuestión de su longitud $(18,8 \mathrm{~m})$ y altura $(5,09)$, según las especificaciones reportadas por la compañía diseñadora (Lockheed Martin Corporation, 2021). La envergadura de esta aeronave es mayor a la del F-22 y esto pasa principalmente por el requerimiento de la misión CAs, la cual exige a la aeronave volar a una menor velocidad que la de crucero de forma segura, por ende, es necesario tener una mayor área alar para así generar la sustentación requerida para esta tarea, y mantener el perfil aerodinámico seleccionado. Esta primicia se puede observar también en el modelo de A-10 Thunderbolt II, una aeronave que, según las especificaciones reportadas por Northrop Grumman, esta aeronave posee 17,42 m de envergadura (Northrop Grumman, 2021).

Se podría entonces calificar como una aeronave pesada, no solo caracterizada por la capacidad de armamento, sino por sus dimensiones. También es posible observar cómo todo el diseño de la aeronave está adaptado a la furtividad, lo que evidencia curvas y superficies que generan ángulos propicios para lograr esta característica.

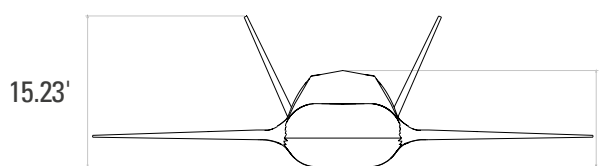

50.52

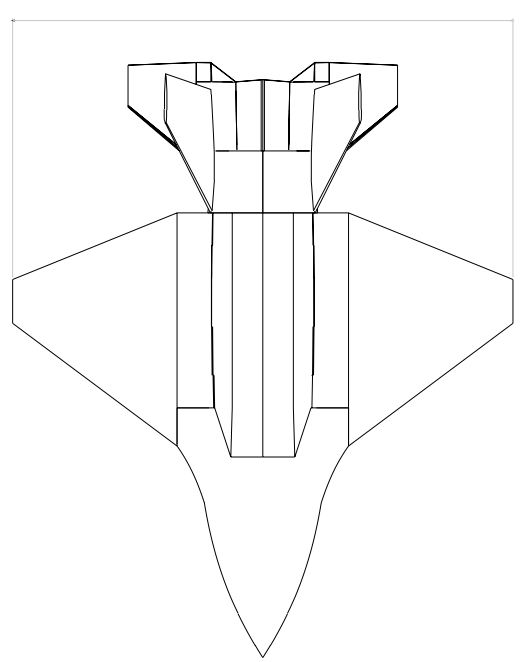

$9.72^{\prime}$

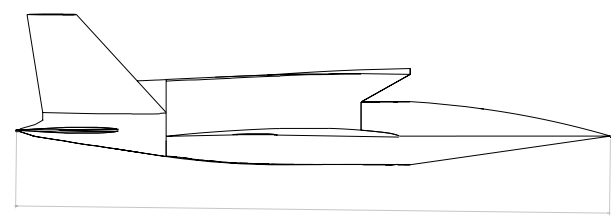

59.98

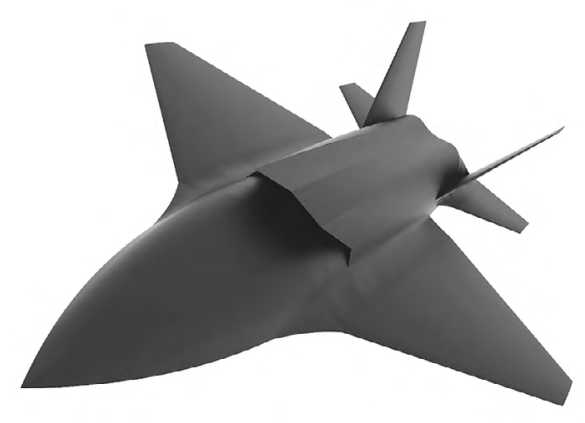

Figura 17. Diseño final de la aeronave para la nueva generación de CAS (dimensiones en ft)

Fuente: elaboración propia. 


\section{Conclusiones}

El diseño planteado estipula una nueva generación de aeronaves CAS para el cumplimiento de misiones de apoyo aéreo cercano, donde por medio de las capacidades tecnológicas especificadas para las aeronaves de combate de quinta generación y no tripuladas, se plantea una visión realista acondicionada a las exigencias de los próximos 20 años de la aviación militar.

Igualmente, el énfasis de furtividad radicado en el diseño de la aeronave se aproxima a las concepciones de la guerra moderna que se plantean para los años venideros, un elemento de alta importancia para la adquisición de nuevas flotas que velen por la soberanía nacional e internacional de cada uno de los países que requieren de este tipo de instrumentos en sus instituciones. Por otra parte, la obtención de un RCs igual a $0,0038 \mathrm{~m}^{2}$ permite conseguir una aeronave de quinta generación de competitividad multilateral en condiciones aerodinámicas y de supervivencia, con cumplimiento de misiones y versatilidad en la operación.

Teniendo en cuenta cada uno de los parámetros estudiados de supervivencia, es importante mencionar que el éxito de esta aeronave frente a misiones de alto riesgo donde se encuentre con múltiples amenazas la beneficia de una manera incondicional, y posibilita que cada una de las selecciones geométricas y estructurales haga parte idónea del diseño final. Además, su distribución alar y conceptualización de fuselaje le permiten adquirir configuraciones limpias manteniendo una capacidad de carga paga especial y no existente en aeronaves de su misma categoría. Cabe agregar que, gracias a su condición de aeronave no tripulada, proporciona una mejora en la toma de decisiones dentro del campo de batalla al reducir el estrés en cabina y salvaguardar la vida del personal durante su operación.

Finalmente, el diseño obtenido demuestra las posibilidades de desarrollo para las próximas generaciones de aeronaves no tripuladas, enfocadas en el comportamiento ante ondas electromagnéticas provenientes de radares. De igual manera, se deja en claro que existen todos los medios para la obtención de aeronaves con bajo RCS, y con las herramientas utilizadas se podría formular una metodología de diseño que tome en cuenta esta área de innovación.

Declaración de conflicto de interés: Los autores no manifiestan conflictos de interés institucionales ni personales.

\section{Referencias}

Atique, M. S. A., Barman, S., Nafi, A. S., Bellah, M., \& Salam, M. A. (2016, 12 de julio). Design of a fifth generation air superiority fighter. AIP Conference Proceedings, 1754. https:// doi.org/10.1063/1.4958444

Airforce Magazine. (2019). B-2 at 30: Improving with Age. Air Force. https://www.airforcemag.com/PDF/Magazine Archive/Magazine\%20Documents/2019/July\%202019/ 0719_B-2\%20for\%20DR.pdf

Au, T. A., Hoek, P. J., \& Lo, E. H. S. (2018). Combat Analysis of Joint Force Options using Agent-Based Simulation. 2018 Military Communications and Information Systems Conference (MilCIS), 1-7. https://doi.org/10.1109/ milcis.2018.8574114

Biswas, K. (2020). Military Aviation Principles. Military Engineering, 1. https://doi.org/10.5772/intechopen.87087

Bravo-Mosquera, P. D., Cerón-Muñoz, H. D., Díaz-Vázquez, G., \& Martini-Catalano, F. (2018). Conceptual design and CFD analysis of a new prototype of agricultural aircraft. Aerospace Science and Technology, 80, 156-176. https:// doi.org/10.1016/j.ast.2018.07.014

Chairman of the Joint Chiefs of Staff (CJCS). (2014). Close Air Support (Joint Publication 3-09.3 ed.). Defense Department Intelligence and Security. https://fas.org/irp/dod dir/dod/jp3_09_3.pdf

Chatzigeorgiadis, F., \& Naval Postgraduate School Monterey CA. (2004). Development of Code for a Physical Optics Radar Cross Section Prediction and Analysis Application. Van Duuren Media.

Chen, S., Yue, K., Hu, B., \& Guo, R. (2015). Numerical Simulation on the Radar Cross Section of Variable-Sweep Wing Aircraft. Journal of Aerospace Technology and Management, 7(2), 170-178. https://dx.doi.org/10.5028/jatm. v7i2.416 
Chen, W. (2004). The Electrical Engineering Handbook. Elsevier Academic Press.

Cidrás-Estévez, J. (2019). Herramienta Informática para el Diseño Conceptual de Aeronaves de Tipo Subsónica y Estudio de las Actuaciones. Universidad de Cádiz. https://rodin. uca.es/xmlui/handle/10498/22567?locale-attribute=es

Correll, J. (2019, 5 diciembre). The Ups and Downs of Close Air Support. Air Force Magazine. https://www.airforcemag. com/article/the-ups-and-downs-of-close-air-support/

Cummings, R. M., \& Schütte, A. (2012). Integrated computational/experimental approach to unmanned combat air vehicle stability and control estimation. Journal of Aircraft, 49(6), 1542-1557. https://doi.org/10.2514/1.C031430

Deskin, W., \& Yankel, J. (2002). Development of the F-22 Propulsion System. 38th AIAA/ASME/SAE/ASEE Joint Propulsion Conference \& Exhibit, 1-10. https://doi.org/10.2514 /6.2002-3624

Sathyamoorthy , D. (2015). A Review of Security Threats of Unmanned Aerial Vehicles and Mitigation Steps. The Journal of Defence and Security, 6(1), 81-97. https://www. researchgate.net/publication/282443666_A_Review_ of_Security_Threats_of_Unmanned_Aerial_Vehicles_ and_Mitigation_Steps

Dirwan, A. (2020). The Analysis of Fighter Aircraft Requirement and Pilot Training for Indonesia's Air Area Security. International Journal of Solid State Technology, 63(3), 1.631-1.632. http://eprints.universitassuryadarma. ac.id/478/1/9_Dirwan.EPrint.pdf

Fields, N. R. (2012). Advantages and challenges of unmanned aerial vehicle autonomy in the Postheroic age [Tesis doctoral. James Madison University]. https://commons. lib.jmu.edu/master201019/205/

Green, J., \& Zeckhauser, R. (2019). Thunder Versus Lightning: A Performance and Cost Analysis of the A-10 "Warthog" Versus the F-35 Joint Strike Fighter. Journal of Benefit-Cost Analysis, 10(3), 434-468. https://doi.org/10.1017/ bca.2019.27

Ho, O. (2018). Future Air Force Close Air Support Aircraft. The Faculty of the Department of Aerospace Engineering San José State University. https://apps.dtic.mil/sti/pdfs/ AD1104495.pdf

Jenn, D. (2019). Radar and laser cross section engineering (3th ed). AIAA Education Series.

James, D. L., \& Gouré, D. (2019). The Implications of Fifth-Generation Aircraft for Transatlantic Airpower A Primer. Atlantic Council. https://www.atlanticcouncil.org/wpcontent/uploads/2019/10/FINAL-Air-Power-DomainReport-WEB-1.pdf
Kong, W., Zhou, D., Yang, Z., Zhang, K., \& Zeng, L. (2020). Maneuver Strategy Generation of UCAV for within Visual Range Air Combat Based on Multi-Agent Reinforcement Learning and Target Position Prediction. Applied Sciences, 10(15), 5198. https://doi.org/10.3390/app10155198

Liangliang, C., Kuizhi, Y., Weigang, G., \& Dazhao, Y. (2016). Integration analysis of conceptual design and stealth-aerodynamic characteristics of combat aircraft. Journal of Aerospace Technology and Management, 8(1), 40-48. https://doi.org/10.5028/jatm.v8i1.514

Lockheed Martin Corporation. (2021). F-22 Raptor Digital, Dominant, Ready. Lockheed Martin Corporation. https:// www.lockheedmartin.com/en-us/products/f-22.html

Nordhagen, L. C. (2018). A-10 Adaptive Basing Operations and Applications in Support of SOF. Naval Postgraduate School. https://apps.dtic.mil/sti/citations/AD1069675

Northrop Grumman. (2021). A-10 Thunderbolt II Specifications. Northrop Grumman. https://www.northropgrumman. com/what-we-do/air/a10-thunderbolt/

Rani, C., Modares, H., Sriram, R., Mikulski, D., \& Lewis, F. L. (2016). Security of unmanned aerial vehicle systems against cyber-physical attacks. Journal of Defense Modeling and Simulation, 13(3), 331-342. https://doi.org/10. $1177 / 1548512915617252$

Raymer, D. P. (2018). Aircraft Design: A Conceptual Approach (Sixth Edition). American Institute of Aeronautics and Astronautics (AIAA).

Sepúlveda-Palacios, E., \& Smith, H. (2019a). Conceptual design of a fifth generation unmanned strike fighter. AIAA Scitech 2019 Forum. https://doi.org/10.2514/6.2019-0811

Sepúlveda-Palacios, E., \& Smith, H. (2019b). Impact of mission requirements on the design of low observable UCAV configurations. Aircraft Engineering and Aerospace Technology, 91(10), 1295-1307. https://doi.org/10.1108/ AEAT-09-2018-0249

Touzopoulos, P., Boviatsis, D., \& Zikidis, K. C. (2018). Constructing a 3D model of a complex object from 2D images, for the purpose of estimating its Radar Cross Section (RCS). Journal of Computations \& Modelling, 1(1), 15-28.

Watts, B. D. (2013). The Evolution of Precision Strike. Center for Strategic and Budgetary Assessments. https://csbaonli ne.org/uploads/documents/Evolution-of-Precision-Stri ke-final-v15.pdf

Yaacoub, J.-P., Noura, H., Salman, O., \& Chehab, A. (2020). Security analysis of drones systems: Attacks, limitations, and recommendations. Internet of Things, 11, 100218. https://doi.org/10.1016/j.iot.2020.100218 